\title{
Comparison of CTA- and DSA-Based Collateral Flow Assessment in Patients with Anterior Circulation Stroke
}

\author{
(DI.G.H. Jansen, (D) O.A. Berkhemer, (D)A.J. Yoo, (D).A. Vos, (D) G.J. Lycklama à Nijeholt, (DM.E.S. Sprengers, (DW.H. van Zwam, \\ (DW.J. Schonewille, (D). Boiten, (D) M.A.A. van Walderveen, (DR.J. van Oostenbrugge, (D)A. van der Lugt, (D).A. Marquering, and \\ (D.B.L.M. Majoie; on behalf of the MR CLEAN investigators (www.mrclean-trial.org)
}

\begin{abstract}
BACKGROUND AND PURPOSE: Collateral flow is associated with clinical outcome after acute ischemic stroke and may serve as a parameter for patient selection for intra-arterial therapy. In clinical trials, DSA and CTA are 2 imaging modalities commonly used to assess collateral flow. We aimed to determine the agreement between collateral flow assessment on CTA and DSA and their respective associations with clinical outcome.
\end{abstract}

MATERIALS AND METHODS: Patients randomized in MR CLEAN with middle cerebral artery occlusion and both baseline CTA images and complete DSA runs were included. Collateral flow on CTA and DSA was graded 0 (absent) to 3 (good). Quadratic weighted $\kappa$ statistics determined agreement between both methods. The association of both modalities with mRS at 90 days was assessed. Also, association between the dichotomized collateral score and mRS 0-2 (functional independence) was ascertained.

RESULTS: Of 45 patients with evaluable imaging data, collateral flow was graded on CTA as 0, 1, 2, 3 for 3, 10, 20, and 12 patients, respectively, and on DSA for $12,17,10$, and 6 patients, respectively. The $\kappa$-value was $0.24(95 \% \mathrm{Cl}, 0.16-0.32)$. The overall proportion of agreement was $24 \%$ $(95 \% \mathrm{Cl}, 0.12-0.38)$. The adjusted odds ratio for favorable outcome on mRS was 2.27 and 1.29 for CTA and DSA, respectively. The relationship between the dichotomized collateral score and mRS 0-2 was significant for CTA $(P=.01)$, but not for DSA $(P=.77)$.

CONCLUSIONS: Commonly applied collateral flow assessment on CTA and DSA showed large differences, indicating that these techniques are not interchangeable. CTA was significantly associated with mRS at 90 days, whereas DSA was not.

ABBREVIATIONS: $C S$ = collateral score; IAT = intra-arterial therapy; MR CLEAN = Multicenter Randomized Clinical Trial of Endovascular Treatment of Acute Ischemic Stroke in the Netherlands

$\mathbf{R}$ ecently, the effectiveness of intra-arterial therapy (IAT) in patients with acute ischemic stroke of the anterior circulation treated within 6 hours after symptom onset was established by

Received March 14, 2016; accepted after revision May 11.

From the Departments of Radiology (I.G.H.J., O.A.B., M.E.S.S., C.B.L.M.M.) and Biomedical Engineering and Physics (H.A.M.), Academic Medical Center, Amsterdam, the Netherlands; Departments of Neurology (O.A.B.) and Radiology (A.v.d.L.), Erasmus MC University Medical Center, Rotterdam, the Netherlands; Texas Stroke Institute (A.J.Y.), Plano, Texas; Departments of Radiology (J.A.V.) and Neurology (W.J.S.), St. Antonius Hospital, Nieuwegein, the Netherlands; Departments of Radiology (G.J.L.N.) and Neurology (J.B.), Haaglanden Medical Center, The Haag, the Netherlands; Departments of Radiology (W.H.v.Z.), and Neurology (R.J.v.O.), Cardiovascular Research Institute, Maastricht, the Netherlands; and Department of Radiology (M.A.A.v.W.), Leiden University Medical Center, Leiden, the Netherlands. H.A. Maquering and C.B.L.M. Majoie shared senior authorship of this article. Paper previously presented at: Annual Meeting of the European Stroke Organization, April 17-19, 2015; Glasgow, Scotland, in the form of an oral presentation by Ivo G.H. Jansen.

Please address correspondence to Ivo G.H. Jansen, MD, Department of Radiology, Gl-230, Academic Medical Center, University of Amsterdam, PO Box 22660, 1100 DD Amsterdam, the Netherlands; e-mail: i.g.jansen@amc.nl

三 Indicates article with supplemental on-line table.

http://dx.doi.org/10.3174/ajnr.A4878 multiple randomized controlled trials. ${ }^{1-5}$ In the Multicenter Randomized Clinical Trial of Endovascular Treatment of Acute Ischemic Stroke in the Netherlands (MR CLEAN), the largest of these trials, a substantial number of patients did not reach functional independence, despite high recanalization rates after IAT. Other trials showed higher recanalization rates, with better overall patient outcome. Contrary to MR CLEAN, these latter trials used neuroimaging for patient selection. In the Endovascular Treatment for Small Core and Anterior Circulation Proximal Occlusion with Emphasis on Minimizing CT to Recanalization Times (ESCAPE) trial, a CTA collateral score (CS) was used for this purpose. ${ }^{3}$

Collaterals are defined as a network of vascular channels that variably restore blood flow when the main supplying artery is blocked. ${ }^{6}$ A good collateral circulation is believed to be of major importance for sustaining the penumbra in patients with acute ischemic stroke, is considered predictive of final infarct volume, and is associated with improved clinical outcome after both intravenous thrombolysis and IAT. ${ }^{7-16}$

The criterion standard for the assessment of collateral flow is multivessel DSA. ${ }^{17}$ This generates images with high spatial and 
temporal resolution, which allows the evaluation of contrast flow into the ischemic region via collaterals. ${ }^{18,19}$ However, in practice, multivessel DSA is considered impractical as a primary diagnostic procedure for acute ischemic stroke triage because speed of treatment is an important factor in these cases. In addition, the expected benefit is low relative to the resources needed. Single-vessel DSA is the pragmatic choice but has several drawbacks, including incomplete assessment of the MCA or anterior cerebral artery territory. Alternatively, CTA is currently used for assessment of collateral flow because of its 24/7 availability and short acquisition time. ${ }^{8-10}$ Also, good interrater agreement for this technique has been reported. ${ }^{10,20}$ Additionally, CTA has the advantage of allowing visualization of collateral flow from all vessels at once, both intra- and extracranial. ${ }^{9}$ A drawback of CTA, however, is the lack of temporal information. Multiphase CTA could solve this problem, but it is not yet widely available.

The purpose of this substudy of MR CLEAN was to assess the agreement between CTA and DSA for grading of collateral flow in patients with acute ischemic stroke due to proximal anterior circulation occlusion, analogous to current practice. In addition, we investigated the association of CTA and DSA collateral grades with clinical outcome as measured on the mRS.

\section{MATERIALS AND METHODS}

\section{Patient Inclusion and Study Design}

The design of MR CLEAN has been published previously. ${ }^{1}$ In short, MR CLEAN was a randomized clinical trial of IAT in addition to the usual care versus usual care alone in patients with a proximal intracranial occlusion in the anterior circulation demonstrated on vessel imaging, treatable within 6 hours after symptom onset. The inclusion criteria for the current substudy were the availability of baseline CTA combined with a complete DSA run of the ipsilateral ICA territory showing a middle cerebral artery occlusion (M1 or M2), including a lateral projection. Exclusion criteria were occlusion of the ipsilateral cervical ICA or ICA terminus, the presence of multiple ipsilateral occlusions, an ipsilateral hypoplastic A1 segment, and occurrence of contralateral flash filling (resulting in dilution of contrast by blood from the contralateral circulation). Approval from the local institutional review board was obtained, and all patients or their legal representatives gave written informed consent to participate. The MR CLEAN study protocol was approved by a central medical ethics committee and the research board of each participating center.

\section{Imaging Assessment}

All imaging data were collected before this substudy, as part of the overall evaluation in MR CLEAN. The relevant scan protocols of CTA and DSA used in this study are shown in the On-line Table. The data were anonymized before assessment. The CS on CTA source imaging was determined in separate sessions by pairs of experienced neuroradiologists, with at least 5 years of experience. Discrepancies between the initial readers were solved by a third reader. CS on DSA was determined by 1 experienced neuroradiologist (A.J.Y., with $>10$ years of experience). All readers were blinded to clinical findings, and the initial readers were blinded to each other's scores. On both CTA and DSA, CS was graded on a 4-point scale, with zero representing absent collateral flow of the
Table 1: Patient characteristics ${ }^{\mathbf{a}}$

\begin{tabular}{ll}
\hline \multicolumn{1}{c}{ Baseline Characteristic } & \multicolumn{1}{c}{ Value } \\
\hline Patients & 45 \\
Median age (range) (yr) & $63(44-85)$ \\
Sex ratio (M/F) & $22 / 23$ \\
Stroke side (L/R) & $22 / 23$ \\
DSA occlusion site & M1 $(n=35), M 2(n=10)$ \\
Clot migration (CTA to DSA) & ICA-T to M1 $(n=1)$ \\
& M1 to M2 $(n=6)$ \\
IVT (yes/no) & M2 to M1 $(n=1)$ \\
Median NIHSS at baseline (range) & $36 / 9$ \\
Median time onset to CTA (range) (min) & $16(6-30)$ \\
Median time onset to DSA (range) (min) & $260(21-496)$ \\
\hline
\end{tabular}

Note:-ICA-T indicates ICA terminus; IVT, intravenous thrombolysis. R, right; L, left. ${ }^{\text {a }}$ Range is displayed in parentheses. IVT displays if patients received IVT prior to IAT. Time to CTA and time to DSA represent the median time in minutes from symptom onset to the acquisition of CTA and DSA scans, respectively.

occluded territory, 1 representing poor collateral flow $(<50 \%$ flow of the occluded territory), 2 representing intermediate collateral flow (between $50 \%$ and $100 \%$ flow of the occluded territory), and 3 representing good collateral flow (100\% flow of the occluded territory). ${ }^{15,21}$

\section{Clinical Outcome Assessment}

The mRS is a 7-point scale on which functional independence of the patient is measured, ranging from 0 (no symptoms) to 6 (dead). In MR CLEAN, the mRS was constructed from a follow-up interview at 90 days, which was conducted by an experienced trial investigator (blinded to the treatment-group allocation) by telephone with the patient, proxy, or health care provider.

\section{Statistical Analysis}

Analysis was performed by using SPSS software (Version 22.0 for Windows; IBM, Armonk, New York). We assessed interobserver reliability for collateral flow on CTA by estimating the agreement beyond chance with quadratic weighted $\kappa$ statistics. Interobserver reliability was considered poor at a $\kappa$-value of 0 , slight between 0.01 and 0.20 , fair between 0.21 and 0.40 , moderate between 0.41 and 0.60 , substantial between 0.61 and 0.80 , almost perfect between 0.81 and 0.99 , and perfect at 1.0. ${ }^{22}$ Quadratic weighted $\kappa$ statistics were also used to determine consistency between both modalities. Ninety-five percent confidence intervals were set to quantify uncertainty. The overall observed proportion of agreement of CS scoring between both methods was determined. Multivariable ordinal logistic regression analysis was performed to determine the adjusted odds ratio of a shift toward better clinical outcome on the mRS, adjusted for early successful recanalization, which was defined as a modified Thrombolysis in Cerebral Infarction score of $2 \mathrm{~B}$ or higher. Additionally, for secondary analyses, CS was dichotomized into poor (absent and poor collateral flow) and good (moderate and good collateral flow), and mRS was dichotomized into a score of $0-2$ (functional independence) versus $3-6 . \chi^{2}$ tests were performed to determine the association between dichotomized CS and mRS 0-2 for both modalities in the entire population and for patients with early recanalization separately. 


\section{RESULTS}

Of the 500 patients in MR CLEAN, 84 met the inclusion criteria on quality of CTA and DSA imaging. Of these, 39 patients were excluded due to the presence of either ICA or ICA terminus occlusion $(n=25)$, presence of multiple occlusions $(n=7)$, occurrence of contralateral flash filling $(n=4)$, a hypoplastic A1-segment $(n=2)$, or motion artifacts $(n=1)$. This exclusion left 45 patients available for comparison. Table 1 provides an overview of the relevant baseline characteristics of the included patients. Thirty-five patients had an M1, and 10, an M2 occlusion on DSA. In 8

Table 2: Distribution of collateral score for CTA and DSA in all patients $^{\mathrm{a}}$

\begin{tabular}{lccccc}
\hline & \multicolumn{5}{c}{ DSA CS } \\
\cline { 2 - 5 } \multicolumn{1}{c}{ CTA CS } & Absent (0) & Poor (1) & Moderate (2) & Good (3) & Total \\
\hline Absent (0) & 2 & 1 & 0 & 0 & 3 \\
Poor (1) & 4 & 3 & 2 & 1 & 10 \\
Moderate (2) & 5 & 8 & 4 & 3 & 20 \\
Good (3) & 1 & 5 & 4 & 2 & 12 \\
Total & 12 & 17 & 10 & 6 & 45 \\
\hline
\end{tabular}

${ }^{a}$ The score corresponding with the relevant category is given in brackets.
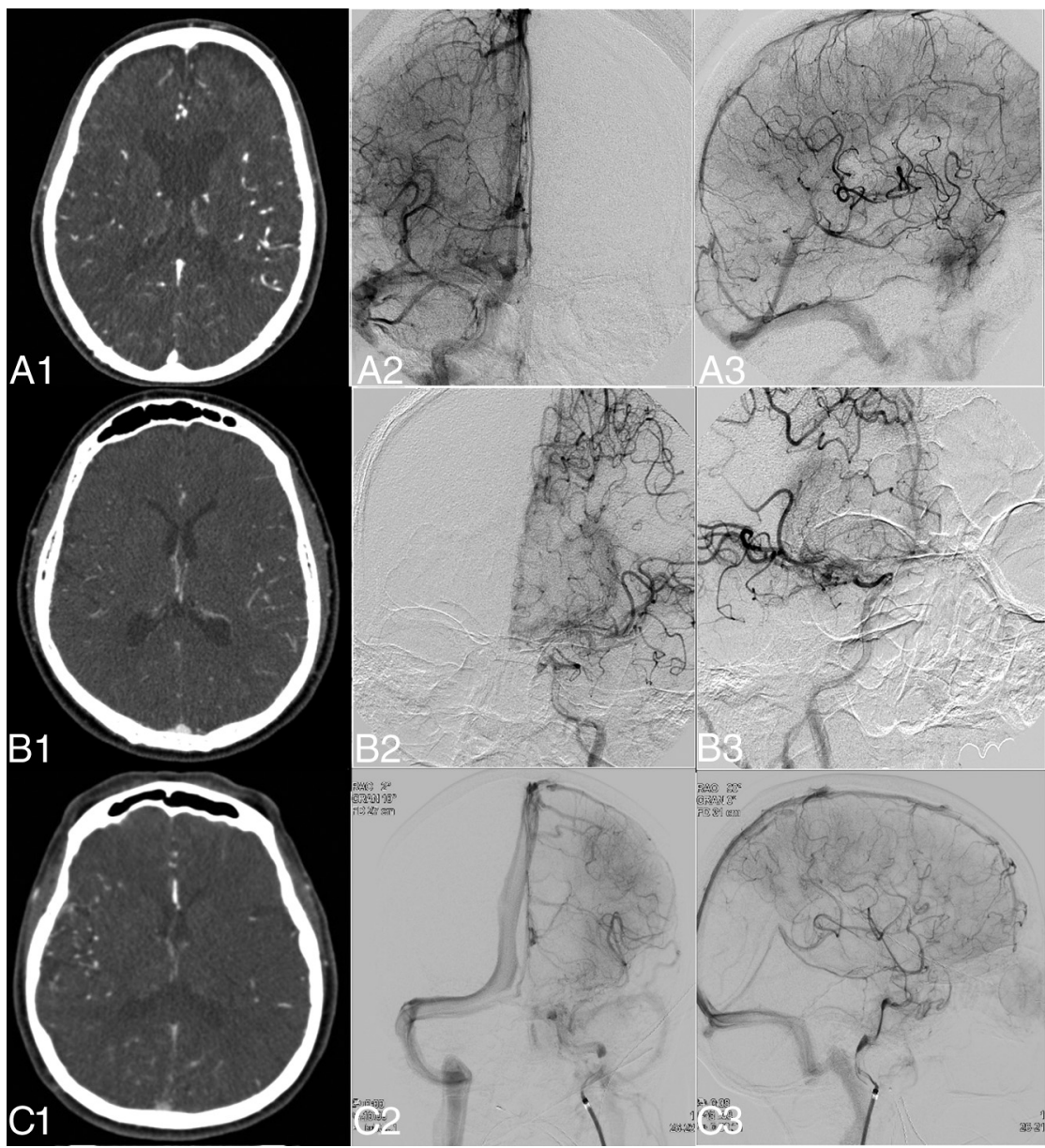

FIG 1. Examples of DSA- and CTA-based collateral scores in 3 different patients. Images were selected by a maximum amount of contrast in the middle cerebral artery for CTA and adequate opacity in the venous phase for DSA. In the left column, the CTA image is shown (Al-Cl); in the middle column, the anteroposterior DSA (A2-C2); and in the right column, the lateral DSA (A3C3). Al-A3, Patient A with a right-sided Ml occlusion, which DSA assessed as grade 3 , and CTA, as grade 3. B1-B3, Patient $B$ with a left-sided M1 occlusion, which DSA assessed as grade 1 , and CTA, as grade 3 . $\mathrm{Cl}-\mathrm{C} 3$, Patient $\mathrm{C}$ with a left-sided M1 occlusion, which DSA assessed as grade 3 collateral flow, and CTA, as grade 1 . of these patients, CTA clot location was different on DSA (in 6 patients from $\mathrm{M} 1$ on CTA to M2 on DSA, in 1 patient from the ICA terminus on CTA to M1 on DSA, and in 1 patient from $\mathrm{M} 2$ on CTA to M1 on DSA). Table 2 shows the distribution of CSs for CTA and DSA in all patients.

Interobserver agreement for collateral flow assessment on CTA had a quadratic weighted $\kappa$-value of 0.68 (95\% CI, $0.40-$ $0.96)$, indicating substantial agreement. Figure 1 gives examples of both methods. The quadratic weighted $\kappa$-value for the agreement between CTA and DSA was 0.24 (95\% CI, 0.16-0.32). When patients in whom clot location differed between CTA and DSA were excluded from analysis, the quadratic weighted $\kappa$-value was 0.25 (95\% CI, 0.13-0.38). The overall observed proportion of agreement was $24 \%$ (95\% CI, 0.12-0.38).

There was a significant shift in $\mathrm{mRS}$ distribution toward better clinical outcome for CTA based on CS (adjusted OR, 2.27; 95\% CI, 1.18-4.40; $P=.015$ ) (Fig 2). For the DSA-based CS, there was no significant association (adjusted OR, 1.29; 95\% CI, 0.76-2.21; $P=.35)$.

Of the 16 cases in which the dichotomized CS was graded as good on DSA (grades 2-3), in 13 (81\%), it agreed with the CTA assessment. In contrast, of 29 cases in which dichotomized CS was graded as poor on DSA (grades $0-1$ ), in only $10(35 \%)$ did it agree with the CTA assessment. There was a significant relationship between dichotomized CS on CTA and mRS 0-2 in the entire population $\left(\chi^{2}=6.7, P=\right.$ $.010)$ and for patients with early recanalization $\left(\chi^{2}=5.2, P=.023\right)$. This was not the case for dichotomized CS on DSA in the total population $\left(\chi^{2}=0.1\right.$, $P=.77)$ or for patients with early recanalization $\left(\chi^{2}=1.1, P=.31\right)$.

\section{DISCUSSION}

With a straightforward grading scale, collateral flow on CTA and DSA has poor agreement, revealing that these approaches are not interchangeable. Irrespective of early recanalization, CTAbased collateral flow is significantly associated with clinical outcome, whereas DSA-based collateral flow is not.

In a recent study, Frolich et $\mathrm{al}^{23}$ found that early triggering of CTA image acquisition could result in diminished visibility and underestimation of collateral flow. In contrast, the same group found that delaying CTA triggering relative to contrast injection could result in overestimation of collateral flow, due to washout of contrast within normal reference arteries, resulting in a relatively weaker opacification of the unaffected 

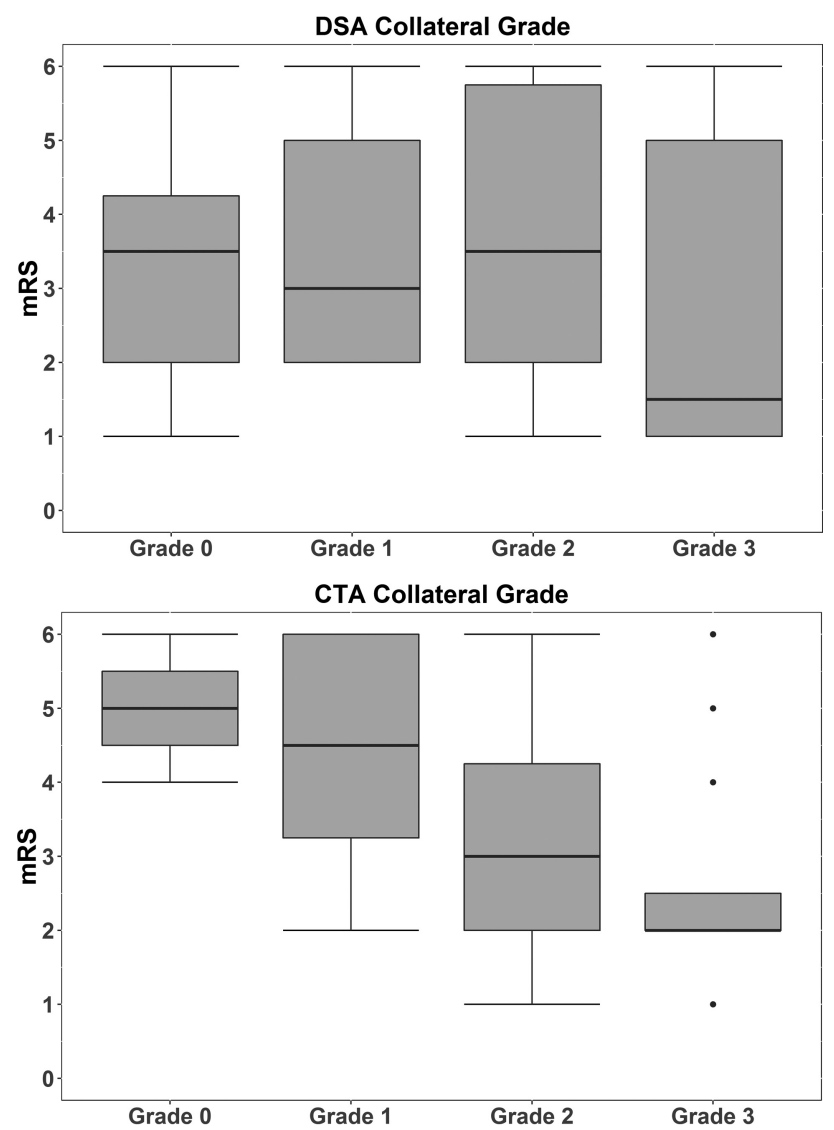

FIG 2. Distribution of CTA- and DSA-based collateral grades on the modified Rankin Scale. For CTA-based collateral grades, a clear change in distribution can be seen toward better clinical outcome for higher collateral grades. For DSA-based collateral grades, there is no change in distribution on mRS for higher collateral grades.

hemisphere. Nambiar et $\mathrm{al}^{13}$ also recently reported this washout effect. Both this over- and underestimation could account for the poor agreement found in our study because CTA only provides a snapshot at a set time point, whereas DSA gives information about collateral flow with time.

In the case of DSA, a complete collateral flow assessment requires imaging of both left and right carotid and 1 or both vertebral arteries. ${ }^{16}$ The delay required for diagnostic DSA and the need for a fast response in acute ischemic stroke management are seen as reasons for not performing DSA collateral imaging routinely in clinical practice, though alternative methods of IAT patient selection on DSA are currently being studied. ${ }^{24}$ Given the potential of collateral flow assessment as a patient-selection tool for IAT, CTA is seen as the imaging technique of choice because it is already obtained for documenting vessel occlusion status, given its availability and low cost. ${ }^{20}$ Another promising alternative is multiphase CTA, as was used for the inclusion of patients with moderate-to-good collaterals in ESCAPE. This also provides fast information on the degree of collateral flow but in a time-resolved manner, without the need for complicated postprocessing. Menon et $\mathrm{al}^{25}$ reported high interrater reliability for this method. Time-invariant CTA based on CTP has also been mentioned and has so far shown good interrater agreement and agreement with DSA. ${ }^{26}$ Kim et $\mathrm{al}^{27}$ compared collateral flow on time-invariant CTA images with DSA and showed a high level of agreement be- tween the 2 modalities, considerably higher than that in the current study. Time-invariant CTA, like DSA and multiphase CTA, has the advantage of giving information of blood flow across time.

There are some limitations to this study. The most important is that validation of DSA as a criterion standard for collateral flow assessment should be based on multivessel imaging. In our study, all patients underwent single-vessel DSA as part of IAT, due to the importance of rapid treatment. Therefore, collateral flow originating from the posterior circulation or the contralateral side could not be assessed, and this omission could have resulted in incomplete information on the degree of collateral flow. ${ }^{13}$ However, because 4-vessel diagnostic DSA is currently not being used routinely, our study is focused on a more pragmatic approach to collateral flow assessment, analogous to current clinical practice. Additionally, our study was designed to minimize this limitation, ensuring, for example, that patients with ICA terminus occlusions or hypoplastic A1 segments were excluded; this exclusion allowed assessment of collateral flow from the ipsilateral anterior and middle cerebral arteries. In addition, in about $20 \%$ of patients, differences in clot location were observed between CTA and DSA, most likely due to clot migration. However when these patients were excluded from the analysis, no difference in the level of agreement between modalities compared with the full analysis was observed. Finally, CTA was performed by using different scanner protocols, due to the large number of centers participating in MR CLEAN. These protocol differences could influence the degree of collateral flow observed. However, we believe that this heterogeneity and the incorporation of cases showing clot migration in our study population add to the generalizability of our study.

Collateral flow could be considered an important target for selection of patients for IAT because it is becoming increasingly clear that patients with poor collateral flow have marginal benefit of treatment. ${ }^{28}$ It can be argued that due to the lack of interchangeability between CTA and DSA, the lack of association between DSA and clinical outcome, and the low application of multivessel DSA in current clinical practice, CTA could be sufficient for this purpose. For future studies excluding patients with poor collateral flow as in ESCAPE, the use of single-vessel DSA for collateral flow assessment could prove precarious. To uphold the role of DSA-based collateral assessment in future patient selection for IAT, further research is warranted to confirm the ability of accurately assessing poor or absent collateral flow on CTA, ideally compared with the criterion standard multivessel DSA. The findings of this study show that the direct comparison of collateral scores acquired by different modalities must be approached with caution. In the near future, it is possible that collateral flow will aid in patient selection for IAT. It is therefore important to consider the properties of the technique with which collateral assessment is performed and how these will affect collateral grading itself, as well as clinical outcome prediction.

\section{CONCLUSIONS}

Commonly applied collateral flow assessment on CTA and DSA showed large differences, indicating that these techniques are not interchangeable. CTA was significantly associated with mRS at 90 days, whereas DSA was not. 


\section{ACKNOWLEDGMENTS}

MR CLEAN investigators: Olvert A. Berkhemer, MD, 1, 2; Puck S.S. Fransen, MD, 2, 3; Debbie Beumer, MD, 2, 4; Lucie A. van den Berg, MD, 5; Hester F. Lingsma, MD, PhD, 7; Albert J. Yoo, MD, 8; Wouter J. Schonewille, MD, 9; Jan Albert Vos, MD, PhD, 10; Paul J. Nederkoorn, MD, PhD, 5; Marieke J.H. Wermer, MD, $\mathrm{PhD}, 11$; Marianne A.A. van Walderveen, $\mathrm{MD}, \mathrm{PhD}, 12$; Julie Staals, $\mathrm{MD}$, $\mathrm{PhD}$, 4; Jeannette Hofmeijer, $\mathrm{MD}, \mathrm{PhD}, 13$; Jacques A. van Oostayen, $\mathrm{MD}, \mathrm{PhD}, 14$; Geert J. Lycklama à Nijeholt, $\mathrm{MD}$, $\mathrm{PhD}, 15$; Jelis Boiten, $\mathrm{MD}, \mathrm{PhD}, 16$; Patrick A. Brouwer, $\mathrm{MD}, 3$; Bart J. Emmer, MD, PhD, 3; Sebastiaan F. de Bruijn, MD, PhD, 17; Lukas C. van Dijk, MD, 18; L. Jaap Kappelle, MD, PhD, 19; Rob H. Lo, MD, 20; Ewoud J. van Dijk, MD, PhD, 21; Joost de Vries, MD, $\mathrm{PhD}$, 22; Paul L.M. de Kort, MD, PhD, 23; Jan S.P. van den Berg, $\mathrm{MD}, \mathrm{PhD}$, 24; Boudewijn A.A.M. van Hasselt, MD, 25; Leo A.M. Aerden, MD, PhD, 26; René J. Dallinga, MD, 27; Marieke C. Visser, $\mathrm{MD}, \mathrm{PhD}, 28$; Joseph C.J. Bot, $\mathrm{MD}, \mathrm{PhD}, 29$; Patrick C. Vroomen, MD, PhD, 30; Omid Eshghi, MD, 31; Tobien H.C.M.L. Schreuder, MD, 32; Roel J.J. Heijboer, MD, 33; Koos Keizer, MD, $\mathrm{PhD}, 34$; Alexander V. Tielbeek, MD, PhD, 35; Heleen M. den Hertog, MD, PhD, 36; Dick G. Gerrits, MD, 37; Renske M. van den Berg-Vos, MD, PhD, 38; Giorgos B. Karas, MD, 39; Ewout W. Steyerberg, MD, PhD, 7; H. Zwenneke Flach, MD, 26; Henk A. Marquering, $\mathrm{PhD}$, 40, 1; Marieke E.S. Sprengers, MD, PhD, 1; Sjoerd F.M. Jenniskens, MD, PhD, 41; Ludo F.M. Beenen, MD, 1; René van den Berg, MD, PhD, 1; Peter J. Koudstaal, MD, PhD, 2; Wim H. van Zwam, MD, PhD, 6; Yvo B.W.E.M. Roos, MD, PhD, 5; Aad van der Lugt, MD, PhD, 3; Robert J. van Oostenbrugge, $\mathrm{MD}, \mathrm{PhD}$, 4; Charles B.L.M. Majoie, MD, PhD, 1; and Diederik W.J. Dippel, MD, PhD, 2.

Affiliations: 1) Department of Radiology, Academic Medical Center, Amsterdam, the Netherlands; 2) Department of Neurology, Erasmus MC University Medical Center, Rotterdam, the Netherlands; 3) Department of Radiology, Erasmus MC University Medical Center, Rotterdam, the Netherlands; 4) Department of Neurology, Maastricht University Medical Center and Cardiovascular Research Institute, Maastricht, the Netherlands; 5) Department of Neurology, Academic Medical Center, Amsterdam, the Netherlands; 6) Department of Radiology, Maastricht University Medical Center, Maastricht, the Netherlands; 7) Department of Public Health, Erasmus MC University Medical Center, Rotterdam, the Netherlands; 8) Department of Radiology, Texas Stroke Institute, Plano, Texas; 9) Department of Neurology, Sint Antonius Hospital, Nieuwegein, the Netherlands; 10) Department of Radiology, Sint Antonius Hospital, Nieuwegein, the Netherlands; 11) Department of Neurology, Leiden University Medical Center, Leiden, the Netherlands; 12) Department of Radiology, Leiden University Medical Center, Leiden, the Netherlands; 13) Department of Neurology, Rijnstate Hospital, Arnhem, the Netherlands; 14) Department of Radiology, Rijnstate Hospital, Arnhem, the Netherlands; 15) Department of Radiology, MC Haaglanden, The Hague, the Netherlands; 16) Department of Neurology, MC Haaglanden, The Hague, the Netherlands; 17) Department of Neurology, HAGA Hospital, The Hague, the Netherlands; 18) Department of Radiology, HAGA Hospital, The Hague, the Netherlands; 19) Department of Neurology, University Medical Center, Utrecht, the Netherlands; 20) Department of
Radiology, University Medical Center, Utrecht, the Netherlands; 21) Department of Neurology, Radboud University Medical Center, Nijmegen, the Netherlands; 22) Department of Neurosurgery, Radboud University Medical Center, Nijmegen, the Netherlands; 23) Department of Neurology, Sint Elisabeth Hospital, Tilburg, the Netherlands; 24) Department of Neurology, Isala Klinieken, Zwolle, the Netherlands; 25) Department of Radiology, Isala Klinieken, Zwolle, the Netherlands; 26) Department of Neurology, Reinier de Graaf Gasthuis, Delft, the Netherlands; 27) Department of Radiology, Reinier de Graaf Gasthuis, Delft, the Netherlands; 28) Department of Neurology, VU Medical Center, Amsterdam, the Netherlands; 29) Department of Radiology, VU Medical Center, Amsterdam, the Netherlands; 30) Department of Neurology, University Medical Center, Groningen, the Netherlands; 31) Department of Radiology, University Medical Center, Groningen, the Netherlands; 32) Department of Neurology, Atrium Medical Center, Heerlen, the Netherlands; 33) Department of Radiology, Atrium Medical Center, Heerlen, the Netherlands; 34) Department of Neurology, Catharina Hospital, Eindhoven, the Netherlands; 35) Department of Radiology, Catharina Hospital, Eindhoven, the Netherlands; 36) Department of Neurology, Medical Spectrum Twente, Enschede, the Netherlands; 37) Department of Radiology, Medical Spectrum Twente, Enschede, the Netherlands; 38) Department of Neurology, Sint Lucas Andreas Hospital, Amsterdam, the Netherlands; 39) Department of Radiology, Sint Lucas Andreas Hospital, Amsterdam, the Netherlands; 40) Department of Biomedical Engineering and Physics, Academic Medical Center, Amsterdam, the Netherlands; and 41) Department of Radiology, Radboud University Medical Center, Nijmegen, the Netherlands.

Data-Monitoring and Safety Board: Chair: Martin M. Brown, National Hospital for Neurology and Neurosurgery, London, United Kingdom. Member: Thomas Liebig, Medizinische Fakultät, University Köln, Germany. Independent Statistician: Theo Stijnen, Leiden University Medical Center, Leiden, the Netherlands.

Advisory Board: Tommy Andersson, neurointerventionist, Karolinska University Hospital, Stockholm, Sweden; Heinrich Mattle, neurologist, University Hospital, Bern, Switzerland; Nils Wahlgren, neurologist, Karolinska Hospital, Stockholm, Sweden.

Research nurses/local trial coordinators: Esther van der Heijden, Naziha Ghannouti, Erasmus MC University Medical Center, Rotterdam, the Netherlands; Nadine Fleitour, Imke Hooijenga, Academic Medical Center, Amsterdam, the Netherlands; Corina Puppels, Wilma Pellikaan, Sint Antonius Hospital, Nieuwegein, the Netherlands; Annet Geerling, Radboud University Nijmegen Medical Center, Nijmegen, the Netherlands; Annemieke Lindl-Velema, Maastricht University Medical Center, Maastricht, the Netherlands; Gina van Vemde, Isala Klinieken, Zwolle, the Netherlands; Ans de Ridder, Paut Greebe, University Medical Center, Utrecht, the Netherlands; José de Bont-Stikkelbroeck, Sint Elisabeth Hospital, Tilburg, the Netherlands; Joke de Meris, MC Haaglanden, The Hague, the Netherlands; Kirsten Janssen, Leiden University Medical Center, Leiden, the Netherlands; Willy Struijk, HAGA Hospital, The Hague, the Netherlands; Tiny Simons, Atrium MC, Heerlen, the Netherlands; Gert Messchendorp, Friedus van der Minne, University Medical Cen-

AJNR Am J Neuroradiol 37:2037-42 Nov 2016 www.ajnr.org 
ter, Groningen, the Netherlands; and Hester Bongenaar, Catharina Hospital, Eindhoven, the Netherlands.

PhD/Medical Students: Silvan Licher, Nikki Boodt, Adriaan Ros, Esmee Venema, Ilse Slokkers, Raymie-Jayce Ganpat, Maxim Mulder, Nawid Saiedie, Alis Heshmatollah, Stefanie Schipperen, Stefan Vinken, Tiemen van Boxtel, Jeroen Koets, Erasmus MC University Medical Center, Rotterdam, the Netherlands; Merel Boers, Emilie Santos, Jordi Borst, Ivo Jansen, Manon Kappelhof, Marit Lucas, Ralph Geuskens, Renan Sales Barros, Roeland Dobbe, Marloes Csizmadia, Academic Medical Center, Amsterdam, the Netherlands.

DISCLOSURES: Albert J. Yoo-UNRELATED: Grants/Grants Pending: Penumbra, ${ }^{*}$ Neuravi, ${ }^{*}$ Comments: Core Imaging Lab activities. Jan Albert Vos-UNRELATED: Grants/Grants Pending: Cordis/Cardinal Health, ${ }^{*}$ Boston Scientific, ${ }^{*}$ Comments: unrestricted departmental educational grants, not related to patients with acute stroke; Payment for Lectures (including service on Speakers Bureaus): Cordis/Cardinal Health, Comments: lectures not related to the subject of patients with acute stroke. Wim H. van Zwam—RELATED: Consulting Fee or Honorarium: Stryker,* Comments: speaker's honorarium (Stryker supports MR CLEAN via funding to the institution). Aad van der Lugt-RELATED: Grant: The MR CLEAN trial was partly funded by the Dutch Heart Foundation and by nominal, unrestricted grants from Angiocare BV, Medtronic/Covidien/ev3, Medac Gmbh/LAMEPRO, Penumbra, Stryker, and Top Medical/Concentric.* Henk A. Marquering-OTHER RELATIONSHIPS: confounder of Nico-lab. Charles B.L.M. Majoie-RELATED: Grant: TWIN Foundation, * Dutch Heart Foundation*; UNRELATED: Payment for Lectures (including service on Speakers Bureaus): Stryker. * Money paid to the institution.

\section{REFERENCES}

1. Berkhemer OA, Fransen PS, Beumer D, et al. A randomized trial of intraarterial treatment for acute ischemic stroke. N Engl J Med 2015; 372:11-20 CrossRef Medline

2. Campbell BC, Mitchell PJ, Kleinig TJ, et al; EXTEND-IA Investigators. Endovascular therapy for ischemic stroke with perfusion-imaging selection. $N$ Engl J Med 2015;372:1009-18 CrossRef Medline

3. Goyal M, Demchuk AM, Menon BK, et al; ESCAPE Trial Investigators. Randomized assessment of rapid endovascular treatment of ischemic stroke. N Engl J Med 2015;372:1019-30 CrossRef Medline

4. Saver JL, Goyal M, Bonafe A, et al; SWIFT PRIME Investigators. Stent-retriever thrombectomy after intravenous t-PA vs. t-PA alone in stroke. $N$ Engl J Med 2015;372:2285-95 CrossRef Medline

5. Jovin TG, Chamorro A, Cobo E, et al; REVASCAT Trial Investigators. Thrombectomy within $\mathbf{8}$ hours after symptom onset in ischemic stroke. N Engl J Med 2015;372:2296-306 CrossRef Medline

6. Faber JE, Chilian WM, Deindl E, et al. A brief etymology of the collateral circulation. Arterioscler Thromb Vasc Biol 2014;34:1854-59 CrossRef Medline

7. Bang OY, Saver JL, Kim SJ, et al. Collateral flow predicts response to endovascular therapy for acute ischemic stroke. Stroke 2011;42: 693-99 CrossRef Medline

8. Menon BK, O'Brien B, Bivard A, et al. Assessment of leptomeningeal collaterals using dynamic CT angiography in patients with acute ischemic stroke. J Cereb Blood Flow Metab 2013;33:365-71 CrossRef Medline

9. Lima FO, Furie KL, Silva GS, et al. The pattern of leptomeningeal collaterals on CT angiography is a strong predictor of long-term clinical outcome in stroke patients with large vessel intracranial occlusion. Stroke 2010;41:2316-22 CrossRef Medline

10. Menon BK, Smith EE, Modi J, et al. Regional leptomeningeal score on CT angiography predicts clinical and imaging outcomes in patients with acute anterior circulation occlusions. AJNR Am J Neuroradiol 2011;32:1640-45 CrossRef Medline
11. Liebeskind DS, Jahan R, Nogueira RG, et al. Impact of collaterals on successful revascularization in Solitaire FR with the intention for thrombectomy. Stroke 2014;45:2036-40 CrossRef Medline

12. Maas MB, Lev MH, Ay H, et al. Collateral vessels on CT angiography predict outcome in acute ischemic stroke. Stroke 2009;40:3001-05 CrossRef Medline

13. Nambiar V, Sohn SI, Almekhlafi MA, et al. CTA collateral status and response to recanalization in patients with acute ischemic stroke. AJNR Am J Neuroradiol 2014;35:884-90 CrossRef Medline

14. Ramaiah SS, Mitchell P, Dowling R, et al. Assessment of arterial collateralization and its relevance to intra-arterial therapy for acute ischemic stroke. J Stroke Cerebrovasc Dis 2014;23:399-407 CrossRef Medline

15. Tan IY, Demchuk AM, Hopyan J, et al. CT angiography clot burden score and collateral score: correlation with clinical and radiologic outcomes in acute middle cerebral artery infarct. AJNR Am J Neuroradiol 2009;30:525-31 CrossRef Medline

16. Liebeskind DS. Collateral circulation. Stroke 2003;34:2279-84 Medline

17. Liebeskind DS, Tomsick TA, Foster LD, et al; IMS III Investigators. Collaterals at angiography and outcomes in the Interventional Management of Stroke (IMS) III trial. Stroke 2014;45:759-64 CrossRef Medline

18. Higashida RT, Furlan AJ, Roberts H, et al; Technology Assessment Committee of the American Society of Interventional and Therapeutic Neuroradiology, Technology Assessment Committee of the Society of Interventional Radiology. Trial design and reporting standards for intra-arterial cerebral thrombolysis for acute ischemic stroke. Stroke 2003;34:e109-37 CrossRef Medline

19. Chng SM, Petersen ET, Zimine I, et al. Territorial arterial spin labeling in the assessment of collateral circulation: comparison with digital subtraction angiography. Stroke 2008;39:3248-54 CrossRef Medline

20. McVerry F, Liebeskind DS, Muir KW. Systematic review of methods for assessing leptomeningeal collateral flow. AJNR Am JNeuroradiol 2012;33:576-82 CrossRef Medline

21. Tan JC, Dillon WP, Liu S, et al. Systematic comparison of perfusion-CT and CT-angiography in acute stroke patients. Ann Neurol 2007;61:533-43 CrossRef Medline

22. Viera AJ, Garret JM. Understanding interobserver agreement: the kappa statistic. Fam Med 2005;37:360-63 Medline

23. Frölich AM, Wolff SL, Psychogios MN, et al. Time-resolved assessment of collateral flow using 4D CT angiography in large-vessel occlusion stroke. Eur Radiol 2014;24:390-96 CrossRef Medline

24. Al-Ali F, Elias JJ, Tomsick TA, et al. Relative influence of capillary index score, revascularization, and time on stroke outcomes from the Interventional Management of Stroke III Trial. Stroke 2015;46: 1590-94 CrossRef Medline

25. Menon BK, d'Esterre CD, Qazi EM, et al. Multiphase CT angiography: a new tool for the imaging triage of patients with acute ischemic stroke. Radiology 2015;275:510-20 CrossRef Medline

26. Smit EJ, Vonken EJ, van Seeters T, et al. Timing-invariant imaging of collateral vessels in acute ischemic stroke. Stroke 2013;44:2194-99 CrossRef Medline

27. Kim SJ, Noh HJ, Yoon CW, et al. Multiphasic perfusion computed tomography as a predictor of collateral flow in acute ischemic stroke: comparison with digital subtraction angiography. Eur Neurol 2012;67:252-55 CrossRef Medline

28. Menon BK, Qazi E, Nambiar V, et al; Interventional Management of Stroke III Investigators. Differential effect of baseline computed tomographic angiography collaterals on clinical outcome in patients enrolled in the Interventional Management of Stroke III Trial. Stroke 2015;46:1239-44 CrossRef Medline 